\section{GAUCHER'S DISEASE OF THE LUNGS}

BY

\section{BERNARD MYERS, C.M.G., M.D., F.R.C.P.}

\section{(With Special Plate)}

Gaucher's disease is comparatively rare, and affection of the lungs by it still more rare. So far I have not been able to find records of a case in this country similar to the one I am describing. Gaucher's disease has been described as a non-hereditary, congenital, familial disease due to changed lipoid metabolism, which produces an abnormal substance called kerasin. More cases occur in females than in males. It is believed that certain cells of the reticulo-endothelial system store the kerasin to form what are called typical Gaucher cells. These cells are chiefly found in the spleen, liver, lymph glands, and bone marrow.

This affection is recognized clinically by the distinct enlargement of the spleen and liver, the pinguecula-like thickenings of the conjunctivae, a light yellowish-brown pigmentation of the skin and sclera, various changes in the bones, leucopenia, thrombocytopenia, and the haemorrhagic diathesis. Sometimes the lymph glands are enlarged. Ascites is only occasionally seen. The pain experienced over the bones particularly is that of a dull ache, but the pain over the spleen and liver, and I believe also in the lungs, is apt to be sharper. The disease may be encountered at any time from birth to about middle age.

Diagnosis is made from the symptoms described above, but especially from the peculiar tint of the skin, splenomegaly, and usually hepatomegaly without ascites, flasklike expansion of the lower ends of the femora accompanied by dull ache or even sharp pain, a raised temperature, leucopenia, and hypochromic anaemia, and is clinched, if necessary, by a splenic puncture and the finding of Gaucher cells, or by finding these cells after biopsy of a gland.

Sobotka and others (1933) state: "At least three pathological entities of generalized 'lipoid storage' disease or lipoidosis have been distinguished from clinical and pathological evidence and confirmed by chemical investigations: (1) Gaucher's splenomegaly, characterized by large deposits of a cerebroside ; kerasin, in spleen and liver; (2) Niemann-Pick's disease, in which the phosphatide and cholesterol contents of the viscera, the bone marrow, and the brain are increased at the expense of neutral fat; (3) Schüller-Christian's disease, with replacement of the bone tissue by cholesterol deposits."

\section{Case History}

A little girl aged 2 years and 7 months was admitted to the Royal Waterloo Hospital on September 16, 1931, on account of a distinctly enlarged abdomen, and general weakness and illness. Upon examination it was found that she was small for her age and appeared to be very ill indeed. There was a slight icteric tint of the skin generally, which also affected the conjunctivae to some extent. The abdominal prominence was due to a greatly enlarged spleen, which reached to the pubes and just to the right of the middle line. Its texture was moderately firm and smooth. The liver was enlarged two fingerbreadths. There had been three attacks of epistaxis during the past few months, the last, a month previously, having been quite severe. The coagulation time of the blood was normal; the bleedingtime a little prolonged -6 minutes. The red blood corpuscles were 800,000 per c.mm. on admission and the haemoglobin 19 per cent., but this was reduced to 16 per cent. two days later. The white blood corpuscles numbered 3,100 . The
Wassermann reaction was negative, and the fragility test normal. The van den Bergh test, both direct and indirect, was negative. The urine was normal. Examination of the stools showed a very slight excess of split fat (fatty acid), but no unsplit fat. No undigested muscle or starch was seen.

The child evidently had had an enlarged abdomen since the early months of life and attended many clinics, etc., and had been apparently regarded as a rare case of rickets. However, no sign of the latter was evident when the child was admitted to hospital. The general appearance, the spleen, the leucopenia, the epistaxis, and anaemia seemed to fit in with the diagnosis of Gaucher's disease, which was made tentatively. It was intended to do a splenic puncture and settle the diagnosis by a microscopical examination of the cells, but on account of the child's desperate condition it was decided not to ; consequently, two days after admission, as a possible chance of saving her life, I asked my colleague, Mr. Rodney Maingot, to perform splenectomy. This was done on September 19, 1931. The spleen weighed 39 oz., and both Dr. Knott and Professor Kettle found typical Gaucher cells present. No similar case had occurred in the family.

Contrary to expectation the little patient made a perfect recovery, and within three to four months the blood count was practically normal.

During April, 1932, she was readmitted to hospital with great pain at the lower end of the right femur. The condition was diagnosed as osteomyelitis, and Mr. St. John Buxton operated and found greyish-green pus from which a few colonies of Staphylococcus albus were grown. Gaucher cells were present in the bony canals of necrosed bone taken away at the operation. Looking back on this case, I am not sure if it was not the ordinary invasion by Gaucher cells which occurs in some of the bones in Gaucher's disease and not osteomyelitis.

During the next two years the patient complained of pain in the back, which was found by $x$-ray examination to be accompanied by gradual atrophy of the bodies of some six or seven dorsal vertebrae, and was obviously due to the Gaucher process. Mr. St. John Buxton undertook to treat the condition.

The following year there was a certain amount of enlargement of the glands in the neck and groin; and pain with a moderately high temperature appeared suddenly, accompanying enlargement of the lower end of the left femur. $X$-ray examination showed the condition to be typical of Gaucher's disease. No operation was done; the temperature and pain gradually disappeared, but the flask-like expanded appearance of the lower end of the left femur remained (as had happened to the right femur).

In August, 1935, the child was again admitted into hospital with what at first sight seemed to be whooping-cough; but on going into the history thoroughly, and taking into consideration the peculiar nature of the cough, the condition appeared to be produced by pressure on the bronchi. Great pain was present behind the sternum, especially the lower end, for which small doses of morphine alone gave relief. The temperature was moderately high, and the respirations and pulse increased in frequency. Careful examination revealed some dullness below the left clavicle and gave the signs of what appeared to be lobar pneumonia. The severe substernal pain gradually disappeared after about a fortnight. Fresh areas of dullness were noted in various parts of the lungs, and the sputum was fairly copious. During this time and the next year the sputum was examined about twenty times for Gaucher cells, but the result was always negative. A number of $x$-ray examinations, made at first fortnightly and afterwards monthly, showed a gradually increasing process spreading from the mediastinum towards each axillary region, the dullness becoming more and more marked. The area of bronchial breathing below the left clavicle persisted, and similar areas gradually became manifest over various parts of the front and back of the lungs. Many medium moist rales were heard, and these persisted. Last November a sudden great pain was felt by the child in the 
right axillary region, and very definite signs of pleurisy were found there.

The blood count in August, 1935, when the child was $6 \frac{1}{2}$ years old, was: red cells, 5,200,000; haemoglobin, 87 per cent. ; colour index, 0.82 ; white cells, 73,600 ; polymorphonuclears, 65 per cent. ; lymphocytes, 27 per cent. In making the differential count the pathologist stated that many dead or basket cells were found. The platelets were fairly numerous.

The pulmonary process was certainly different from anything I had seen in a child before, and as Gaucher's disease was present in other parts of the reticulo-endothelial system the pulmonary condition was looked upon as being probably due to the same affection.

During 1935 and 1936 the liver became greatly enlarged and reached nearly to the pubes, also extending beyond the umbilicus into the left hypochondriac region. The child's growth was certainly being interfered with by the faulty fatty metabolism present. She was distinctly smaller and much lighter in weight than her sister, who was two years younger. During 1936 various nervous manifestations became evident; she would become irritable without cause, and developed movements of the eyes, mouth, nose, face, and neck, often for only a few days at a time, but they never entirely disappeared.

Death took place on January 15, 1937, and as a partial necropsy was allowed the diagnosis of Gaucher's disease was verified. I append reports.

\section{Pathological Report}

The following are the necropsy findings reported by Dr. F. A. Knott:

The body showed considerable wasting and, for the child's age, underdevelopment of the muscles and bony skeleton. The dorsal vertebrae were markedly arched forward owing to the collapse of some of their bodies. The abdomen was very protuberant because of the greatly enlarged liver. There were no bruises or pigmentation, and no oedema.

On opening the abdomen the most striking feature was an enormously enlarged liver, which weighed $3 \mathrm{lb} .7 \mathrm{oz}$. Its surface was smooth, and the enlargement occurred equally throughout all the lobes. The gall-bladder and biliary tracts were normal. The liver had a pale pink colour as a whole, with irregular patches of dull yellow. It had formed no adhesions.

The intestines showed no abnormality, and the kidneys and genito-urinary tract appeared normal, but there was considerable enlargement of practically all the abdominal glands, this being more marked in the upper than in the lower abdomen.

When the thorax was opened the heart and great vessels were found to be normal, except that they were variously displaced through enlargement of the mediastinal glands, the enlargement continuing into the root of each lung. The pulmonary appearances are described below. There were no pleural effusions or adhesions. The thymus, thyroid, larynx, and pharynx were normal; the brain was not examined.

The spleen had already been removed about five and a half years previously, and at that time weighed $2 \mathrm{lb} .7 \mathrm{oz}$. Its general and histological appearances had been reported as typical of a Gaucher spleen.

Specimens for section were taken from the liver, from glands in various parts of the body where noticeable enlargement had occurred, from a rib, a part of the sternum, the root of a lung, a small solidified area in the lung, the kidney, the pancreas, and heart muscle.

Large masses of Gaucher cells were found in every instance of glandular enlargement, and in all the samples of bone marrow examined; the kidney, pancreas, and heart muscle were free. In the liver enormous collections of these cells appeared, pushing aside the normal liver tissue, which on the whole had undergone little degeneration. In sharp contrast, owing to its location inside the bones, the bone marrow was very largely compressed and displaced, and the total functioning marrow must have been much below normal. The changes in the bodies of the vertebrae were due to Gaucher deposition and pressure damage.

In short, the characteristic appearances were that at all possible sites reticulo-endothelial deposition of Gaucher substance had taken place with compression and displacement of neighbouring cells, but without producing further damage than would arise from compression.

The immediate cause of death was to be found in a simple terminal, mainly basal, bronchopneumonia; but the findings in the lungs had an interest beyond this, because they had clearly been involved in the primary Gaucher disease before the final infection took place.

\section{COMMENTARY ON THE FINDINGS}

This case illustrates particularly well the reasons for classifying Gaucher's disease among the reticulo-endothelioses. Not only were the liver, spleen, larger lymph glands, and the bone marrow packed with large masses of typical cells containing the non-doubly refractive Gaucher substance, but collections of these cells could be seen wherever lymph nodes occurred.

This process was found to account for the early physical signs noted in the patient's chest long before there was any indication of bronchopneumonia. At the post-mortem examination the mediastinal and hilum glands were all grossly enlarged and contained masses of Gaucher "cells, with the result that the roots of both lungs were apparently extensively "invaded" by the disease, as seen by the increased root shadows in the radiographs and by the physical signs.

But another feature was noticeable, particularly in the upper lobes. In many of the small subserous and interlobular lymph nodes the reticulo-endothelial cells were loaded with Gaucher substance, producing a series of small apparently solidified areas, the neighbouring air spaces being markedly compressed. In between the compressed areas the lung tissue was relatively normal, the only change being the very recent terminal, mainly basal, bronchopneumonia. There was no evidence of old or recent tubercle, and the changes first noted clinically in the lungs were undoubtedly due to deposition of the Gaucher material, even in such outlying portions of the reticulo-endothelial system as the distal lymphatic system of the lungs.

\section{Review of the Literature}

Although, as I have stated, I have not succeeded in finding records of a case of Gaucher's disease affecting the lungs in England, one has been published in France by Merklen, Waitz, and Warter (1933) on the "maladie de Gaucher," in which typical Gaucher cells were found in the sputum. They state: "L'expectoration permanente pendant plusieurs mois de crachets muqueux, l'abondance de cellules constituant parfois des amas considérables, la proportion notable et constante de cellules de Gaucher typiques nous permettant de décrire une forme pulmonaire 
de la maladie de Gaucher." The above was a typical case of Gaucher's disease affecting the spleen, liver, femora, and the vertebral column. Epistaxis occurred; the patient was 51 years of age; and anaemia was present along with leucopenia.

Although we repeatedly examined the sputum of our own patient, we never discovered Gaucher cells in it ; still, the case quoted above shows that such can occur, and they therefore should be looked for.

I would like to refer here to "Studies on the Chemistry of Gaucher's Disease: An Analysis of Three Spleens," by R. H. S. Thompson and G. Payling Wright (1937). They state "that the essential pathological change is a specific storage of kerasin. Concerning the origin of this kerasin or its mode of accumulation in the reticulo-endothelial cells throughout the body nothing at present is known, but two hypotheses may be suggested for the aetiology of the condition. In the first place the distended 'Gaucher cells' may be merely the result of an absorptive action of the histiocytes on an increased blood kerasin." They continue: "An alternative theory, based on the assumption that the galactolipins are possible important intermediaries in the antibody responses of the reticulo-endothelial system, suggests itself as the result of recent work on certain aspects of immunological chemistry." They state, further : "In view of this work, therefore, it is conceivable that the galactolipins, whose physiological role in the body is at present unknown, may play a part in the production of antibodies by the reticulo-endothelial cells, and that Gaucher's disease may be some 'inborn error of metabolism' of the mechanism responsible for some stage in the antibody production of the body, whereby excessive quantities of kerasin are synthesized and stored by the reticulo-endothelial cells."

\section{Summary}

A case of typical Gaucher's disease in a little girl is described in which the spleen, liver, both femora, some of the dorsal vertebrae, lymph glands, and the lungs were affected.

The affection of the lungs by this disease must be extremely rare, for the only other definite case so far noted is quoted in the French literature.

The most recent views on the pathological chemistry of Gaucher's disease are stated.

In the Special Plate photomicrographs are reproduced of sections of the liver (Fig. 1), a lymphatic gland (Fig. 2) the bone marrow of a rib (Fig. 3), the spleen (Fig. 4), and the lung (Fig. 5). Fig 6 is a reproduction of a radiograph taken four months before death and showing clearly the extension of the Gaucher process from the mediastinum outwards and to the apices. Ludwig Pick (1933) states that in fresh specimens the Gaucher cells are homogeneous, opaque, and dull hyaline in appearance. With the Mallory stain, with acid-fuchsine, and aniline-blue-orange $G$, and with previous fixation with picric acid and ammonium bichromate, they appear a light blue, and under higher magnification show, in their cytoplasm, an irregular network, bound like spider webs with the finest thread-like fibrils. Through these the cytoplasm appears wrinkled rather than foamy or roundly vacuolated. The wrinkles correspond to the remains of the spongioplasm in the meshes of which the Gaucher substance is stored. Large cells with this type of cytoplasm are found in no other affection. The cells measure from 20 to $80 \mu$ in their largest diameter; they are polymorphous, and are often furnished with projections. The nuclei are either single or multiple, and not infrequently are quite numerous (there were twenty-one in one cell). They are mostly pyknotic. In those cells where there is a single: nucleus it is often eccentric in position, generally near the cell border. Mitoses are disputed. Pick found arterial branches within the nests of Gaucher cells.
REFERENCES

Merklen, P., Waitz, R., and Warter, J. (1933). Ann. de Méd., $33,97$.

Pick, Ludwig (1933). Amer. J. med. Sci., 185, 453.

Sobotka, H., et al. (1933). Biochem. J., 27, 2031.

Thompson, R. H. S., and Wright, G. Payling (1937). Guy's Hosp. Rep., 87, 30 .

\section{TEMPORARY POSTPONEMENT OF MEN- STRUATION BY OESTRADIOL BENZOATE}

BY

GEORGE L. FOSS, M.A., B.Ch.Camb.

Colston Research Fellow; Clinical Assistant to the Gynaecological Department, Bristol Royal Infirmary

\section{(With Special Plate)}

There are occasions when a temporary postponement of menstruation would be a great advantage. I refer to such times as athletic championships, examinations, and weddings, when a condition of bodily comfort and peace of mind is essential, and this cannot be if these events coincide with menstruation. In such cases I suggest that the delay of menstrual bleeding for a few days or a week is a justifiable therapeutic measure on a few special occasions of a woman's life; but I do not think it is a practice to be carried out more than once or twice during an individual lifetime, as it may be unwise to upset the normal delicate pituitary-ovarian rhythm too frequently.

The idea of such therapy arose from the treatment of some of a series of cases with oestradiol benzoate.* It was found that if injections were carried out through the whole cycle the next bleeding was delayed or even inhibited. In some cases of oligomenorrhoea and hypomenorrhoea treatment was sometimes followed by a short period of amenorrhoea. In such cases bleeding might be delayed if injections of 10,000 I.B.U. were repeated, whereas in patients with normal loss this occurred if dosage was raised to 50,000 I.B.U. This is explained by Zuckerman's work (1936) on the production of uterine bleeding by oestrin withdrawal. Cyclical treatment of cases of oligomenorrhoea was not followed by amenorrhoea when 50,000 I.B.U. were given only in the first fifteen days of the cycle.

Zuckerman (1936), working with monkeys, showed definitely that if oestrone is given for a period of fifteen days in a dosage known to be adequate for proliferation, and is then withdrawn, bleeding will occur about seven to eight days later. If this threshold dose is continued throughout the cycle bleeding is postponed for as long as the dose is kept above threshold. If allowed to drop below, either by reducing dosage or by increasing the interval between injections, bleeding would occur. Zuckerman (1936) suggested that if - superthreshold doses were given throughout the cycle ovulation was inhibited and the proliferation phase was continued. If injections were started after the middle of the cycle following ovulation it was doubtful whether oestrone would then inhibit bleeding. Evidence was inconclusive on this point even from Zondek's series (1936). If given after the middle of the cycle it was necessary to use much larger doses to cause inhibition. B. Zondek (1936), in the series referred to, noticed that during therapeutic enlargement of hypoplastic uteri of sterile women the next bleeding was often delayed if moderate doses were continued through the

* Oestradiol is six times as physiologically active as oestrone. The standardized preparation used in this work was progynon B oleosum-Schering. 


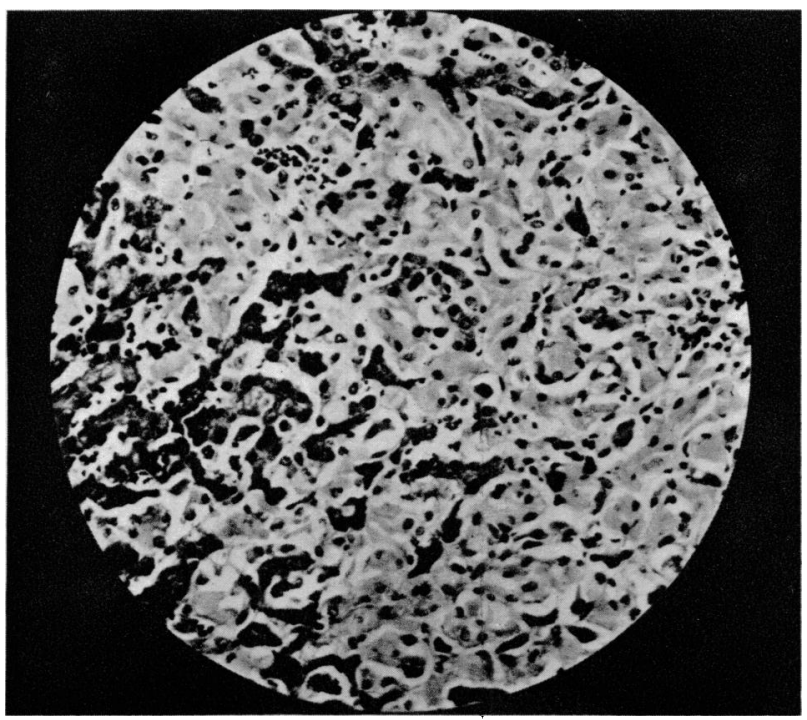

Fig. 1.-Section of liver.

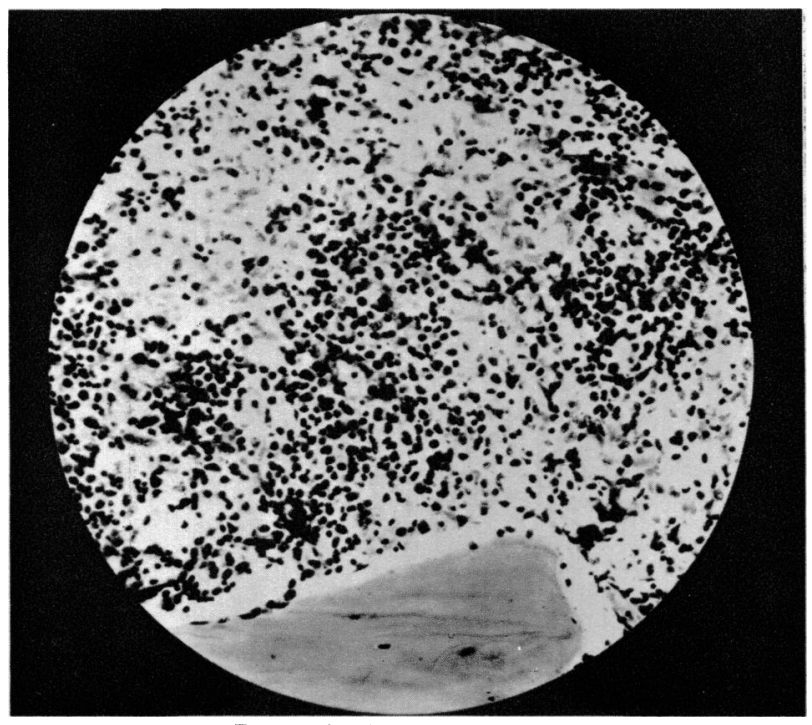

Fig. 3.- Section of bone marrow

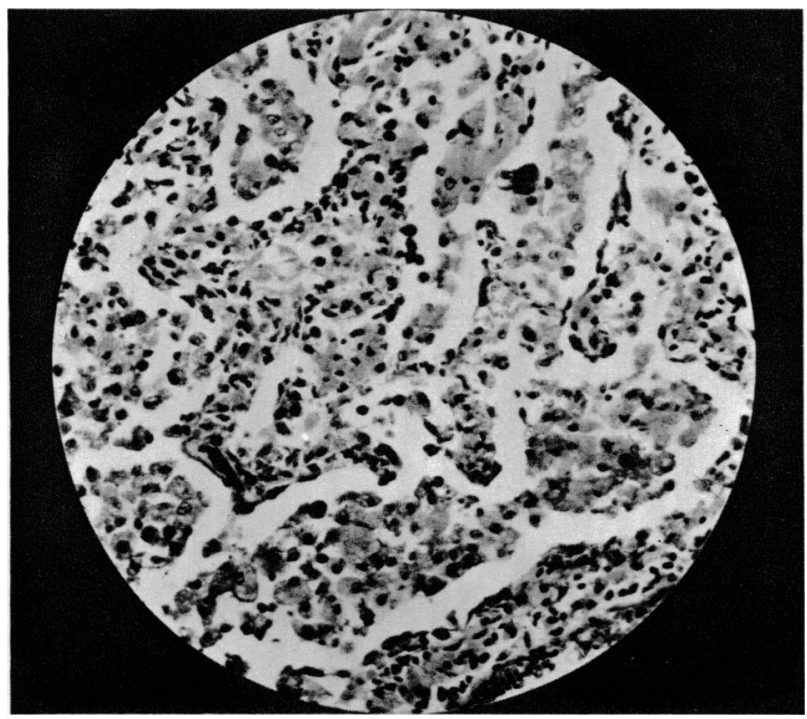

Fig. 5.-Section of lung, showing Gaucher cells occluding the alveoli.

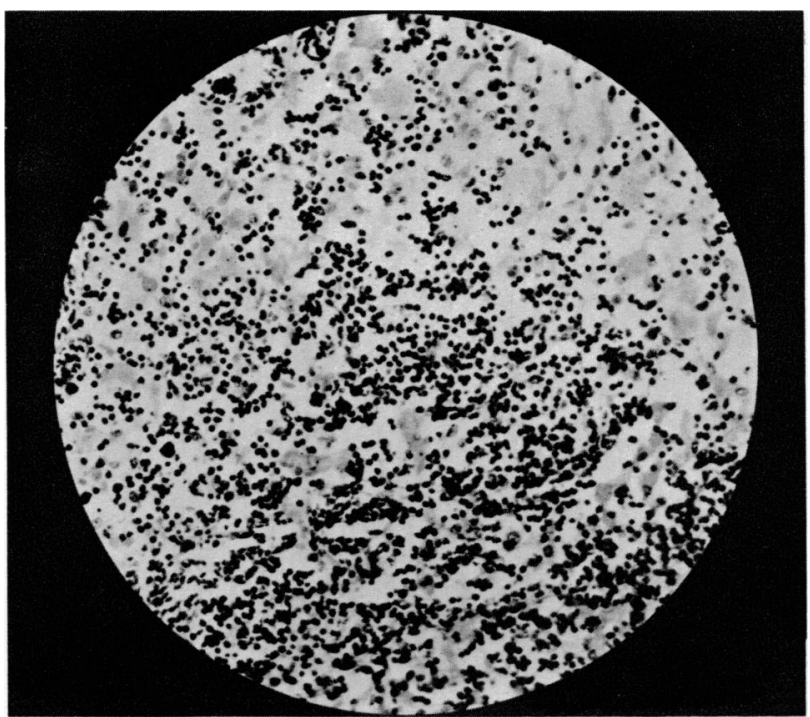

Fia. 2.-Section of a lymph gland.

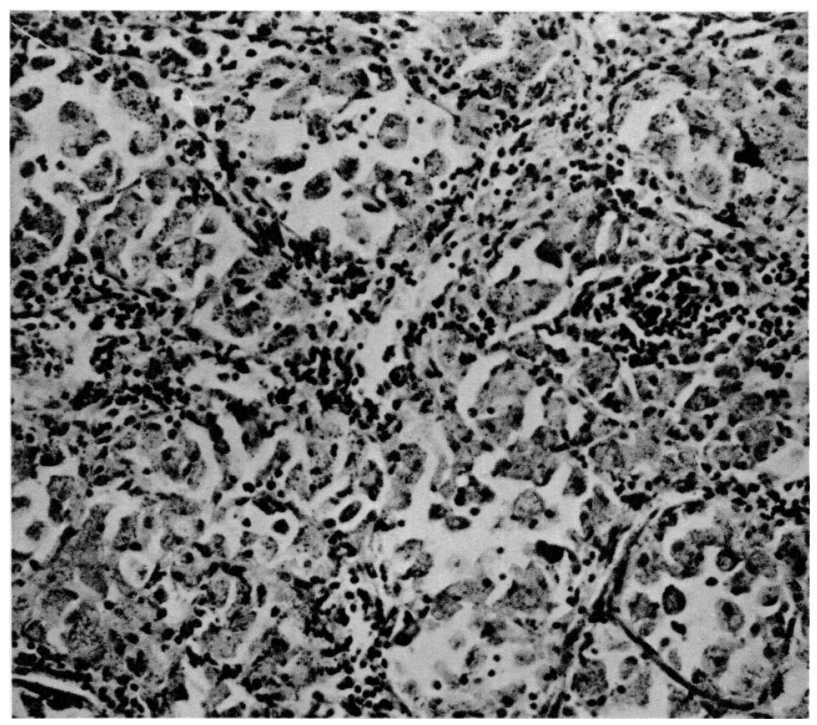

Fif. 4.-Section of spleen, showing nests of Gaucher cells.

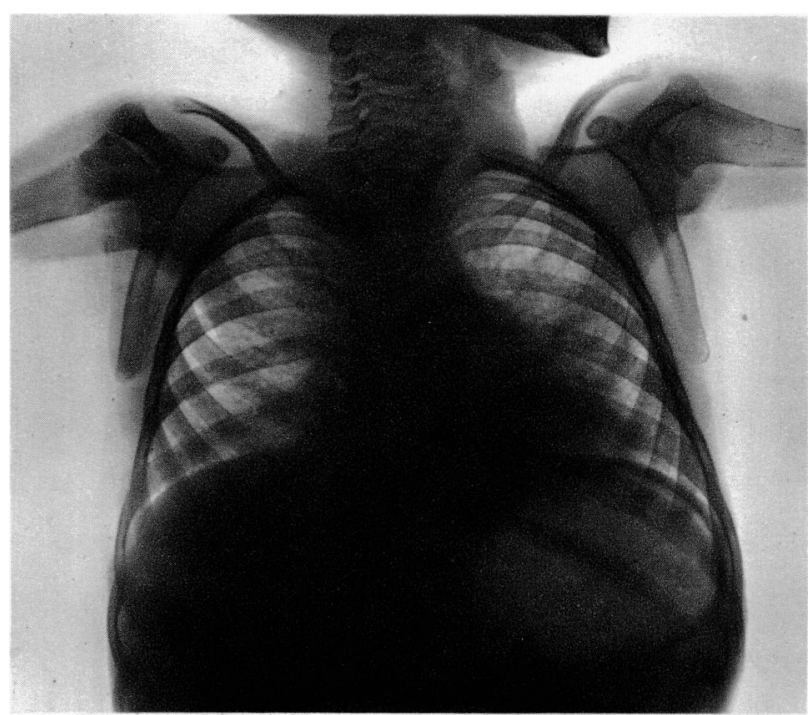

Fia. 6. - A radiograph of the lungs (26/8/35), showing the extension the apices. This was taken just four months before death. 